\title{
Analysis of the aplyronine A-induced protein-protein interaction between actin and tubulin by surface plasmon resonance
}

\author{
Yuichiro Hirayama, ${ }^{a, \dagger}$ Kota Yamagishi, ${ }^{a}$ Tomohiro Suzuki,, ,

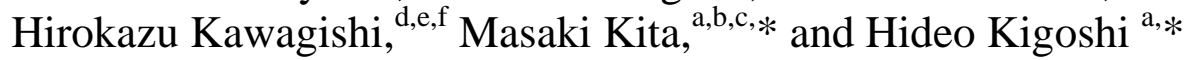 \\ ${ }^{a}$ Graduate School of Pure and Applied Sciences and ${ }^{b}$ Tsukuba Research Center for Interdisciplinary Materials Science (TIMS), \\ University of Tsukuba, and ${ }^{c}$ JST-PRESTO, 1-1-1 Tennodai, Tsukuba 305-8571, Japan \\ ${ }^{d}$ Research Institute of Green Science and Technology, ${ }^{e}$ Graduate School of Agriculture, and ${ }^{f}$ Graduate School of Science and Technology,
} Shizuoka University, 836 Ohya, Suruga, Shizuoka 422-8529, Japan

Received XX YY 20ZZ; revised XX YY 20ZZ; accepted XX YY $20 Z Z$

\begin{abstract}
The antitumor macrolide aplyronine A induces protein-protein interaction (PPI) between actin and tubulin to exert highly potent biological activities. The interactions and binding kinetics of these molecules were analyzed by the surface plasmon resonance with biotinylated aplyronines or tubulin as ligands. Strong binding was observed for tubulin and actin with immobilized aplyronine A. These PPIs were almost completely inhibited by one equivalent of either aplyronine A or C, or mycalolide B. In contrast, a non-competitive actindepolymerizing agent, latrunculin A, highly accelerated their association. Significant binding was also observed for immobilized tubulin with an actin-aplyronine A complex, and the dissociation constant $K_{\mathrm{D}}$ was $1.84 \mu \mathrm{M}$. Our method could be used for the quantitative analysis of the PPIs between two polymerizing proteins stabilized with small agents. (c) 2016 Elsevier Science. All rights reserved
\end{abstract}

\section{Introduction}

Aplyronine A (1) is a 24-membered macrolide that was isolated from the sea hare Aplysia kurodai (Figure 1). ${ }^{1-3}$ It bears a C24-C34 side chain moiety that includes a terminal $N$-methyl enamide group and two amino acid esters ( $N, N, O$-trimethylserine and $N, N$-dimethylalanine) as structural features. Aplyronine A exhibits strong cytotoxicity against HeLa S3, a human cervical carcinoma cell line $\left(\mathrm{IC}_{50} 10 \mathrm{pM}\right)$, and potent antitumor activities against several murine xenograft models. Previous studies have shown that aplyronine A (1) inhibits actin polymerization by forming a 1:1 complex with the globular monomeric molecule. ${ }^{4}$ X-ray analysis of the actinaplyronine A complex revealed that $\mathbf{1}$ intercalates into the hydrophobic cleft between subdomains (SD) 1 and 3 of actin by using its side chain. ${ }^{5}$

Recent target identification studies using the photoaffinity derivatives and other biochemical

Keywords: surface plasmon resonance; protein-protein interaction; antitumor natural products; actin; tubulin.

* Corresponding authors. e-mail: mkita@ chem.tsukuba.ac.jp (M.K.), kigoshi@chem.tsukuba.ac.jp (H.K.)

$\uparrow$ Present address: Department of Pharmaceutical Sciences, University of Shizuoka, Shizuoka 422-8526, Japan.

$\ddagger$ Present address: Center for Bioscience Research and Education, Utsunomiya University, Tochigi 321-8505, Japan. experiments have established that aplyronine A (1) synergistically binds to tubulin in association with actin. ${ }^{6}$ Several tubulin-targeting natural products, which regulate spindle microtubule dynamics, have been widely used in cancer chemotherapy. To our knowledge, aplyronine A (1) is the first molecule to be known to promote the proteinprotein interactions (PPIs) between tubulin and actin to exert highly potent biological activities. ${ }^{7,8}$ However, little is known about their intermolecular interactions and binding kinetics. Several biochemical techniques that can be used to discover and characterize PPI modulators have been established; e.g., enzyme-linked immunosorbent assays (ELISA), fluorescence resonance energy transfer (FRET) and related proximity-based methods, and surface plasmon resonance (SPR). ${ }^{9,10}$ To further analyze the binding kinetics and stabilizing effect of $\mathbf{1}$ for the PPIs between actin and tubulin, in the present study we conducted a biosensor SPR analysis of aplyronines.

\section{Results and Discussion}

2.1. Preparation of aplyronine biotin derivatives and their interactions with actin

To immobilize aplyronines on a streptavidin sensor chip, we used their biotin derivatives. The PEG-linked aplyronine A biotin conjugate $\mathbf{3}$ has been used for target identification studies. ${ }^{11}$ We also used aplyronine C (2), a natural congener of $\mathbf{1}$ that lacks the $\mathrm{C} 7$ trimethylserine ester moiety, as a negative control. ${ }^{12,13}$ It exhibits as much actin- 
depolymerizing activity as $\mathbf{1}$, but is 1,700 times less cytotoxic due to its inability to interact with tubulin. Thus, we prepared an aplyronine $\mathrm{C}$ biotin derivative $\mathbf{4}$ from $\mathbf{2}$ (Figure S1). Aplyronine $\mathrm{C}$ analog 4 was found to be 7,300 times less cytotoxic against HeLa S3 cells than $3\left(\mathrm{IC}_{50}=\right.$ $700 \mathrm{nM}$ ), which reflected the significant difference in cytotoxicity between the natural aplyronines.

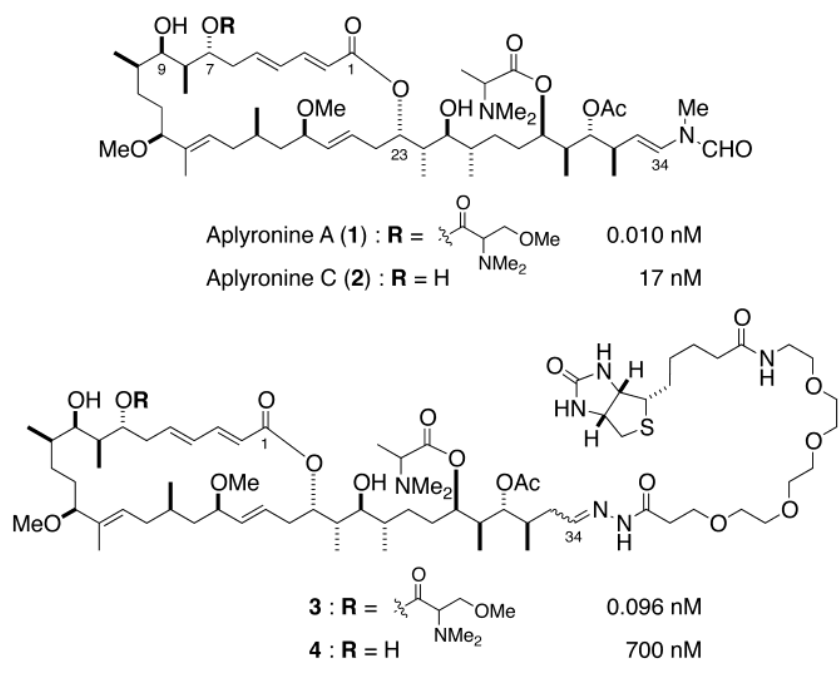

Figure 1. Structures of aplyronines and their biotin derivatives. $\mathrm{IC}_{50}$ values for cytotoxicity against HeLa S3 cells are shown below each compound (to the right).

To investigate the interactions between actin and aplyronines, biotin derivatives $\mathbf{3}$ and $\mathbf{4}$ were used as ligands for SPR analysis. Strong binding was observed for actin with ligand $\mathbf{3}$ on the surface (Figure 2A). The equilibrium constants obtained from global kinetic fitting of the sensorgrams were then fitted to an approximate 1:1 model (Figure S2). The on-rate constant $k_{\mathrm{a}}$ was determined to be $4.54 \times 10^{3} \mathrm{M}^{-1} \mathrm{~s}^{-1}$, while the off-rate constant $k_{\mathrm{d}}$ was $0.0183 \mathrm{~s}^{-1}$, which makes this ligand $\mathbf{3}$ a moderate actin binder in this set $\left(K_{\mathrm{D}} 4.02 \times 10^{-6} \mathrm{M}\right)$ (Table 1$)$. Under the same conditions, ligand 4 bound to actin with a similar offrate constant $k_{\mathrm{d}}$ as $\mathbf{3}$, but with an approximately five-hold smaller on-rate constant $k_{\mathrm{a}}$, which led to a substantial decrease in association $\left(K_{\mathrm{D}} 1.80 \times 10^{-5} \mathrm{M}\right)$. The $K_{\mathrm{D}}$ value for actin and 1 has been reported to be $1.0 \times 10^{-7} \mathrm{M}$, based on a fluorescent actin-polymerization inhibitory assay. ${ }^{4}$ These decreases in affinity with actin might be due to modification of the side chain moiety in $\mathbf{3}$.

Table 1. Binding kinetics of immobilized aplyronines-actin interaction

\begin{tabular}{cccc}
\hline ligand & $k_{\mathrm{d}}\left(\mathrm{s}^{-1}\right)$ & $k_{\mathrm{a}}\left(\mathrm{M}^{-1} \mathrm{~s}^{-1}\right)$ & $K_{\mathrm{D}}(\mathrm{M})$ \\
\hline $\mathbf{3}$ & $1.83 \times 10^{-2}$ & $4.54 \times 10^{3}$ & $4.02 \times 10^{-6}$ \\
$\mathbf{4}$ & $1.51 \times 10^{-2}$ & $8.38 \times 10^{2}$ & $1.80 \times 10^{-5}$ \\
\hline
\end{tabular}

${ }^{a}$ Values were determined by global fitting of five concentrations of analyte (actin) as shown in Figure 2A.

\subsection{Interactions of actin and tubulin with immobilized aplyronine A}

Next, interactions of actin and tubulin with immobilized aplyronine A were analyzed by SPR. By using biotinylated aplyronine A as a ligand, actin and tubulin were simultaneously injected. However, no meaningful SPR responses were observed despite the strong interactions between actin and immobilized aplyronine $\mathrm{A}$ as mentioned above. After several attempts, however, strong binding was observed for tubulin and actin with immobilized aplyronine A when actin was loaded in advance and actin and tubulin were continuously co-injected (Figure 2B). Tubulin binding was concentration-dependent (0 to $2 \mu \mathrm{M})$ (Figure S3). On the other hand, no significant binding was observed when tubulin alone was injected over the actin-preloaded surface. Since actin is unstable in the tubulin-stabilizing PIPES$\mathrm{KCl}$ buffer supplemented in the running buffer for the second injection, preloaded actin might rapidly dissociate from the surface or lose the ability to bind to tubulin via aplyronine $\mathrm{A}$.

To demonstrate the binding specificity of PPIs between actin and tubulin, various actin-targeting agents were coinjected with actin and tubulin (Figure 2C). Mycalolide B is an actin-depolymerizing agent that interacts with the same binding site of actin as aplyronines. ${ }^{14}$ The $K_{\mathrm{D}}$ value for actin and mycalolide B has been reported to be 13-20 $\mathrm{nM} \cdot{ }^{15,16}$ As expected, one equivalent each of aplyronines A (1) and C (2) and mycalolide B almost completely inhibited the interaction of tubulin with the actin-aplyronine A complex on the surface. In contrast, an excess amount of latrunculin A, another actin-depolymerizing agent that binds to the ATP-binding site of actin (opposite site for aplyronines), highly enhanced the association of actin and tubulin onto the aplyronine A-immobilized chip (ca. 70\% increase in RU). Previous ultracentrifugation experiments and gel-permeation HPLC analysis suggested that monomeric actin assembles and loses its function under high $\mathrm{Mg}^{2+}$ conditions. ${ }^{6}$ In fact, the loading of actin in the PIPES-KCl-enriched buffer increased the bulk response, presumably due to the unspecific binding of actin oligomers (Figure 2B). Thus, latrunculin A might increase the effective concentration of monomeric actin in the tubulin-stabilizing buffer, which significantly enhanced the formation of the actin-aplyronine A-tubulin ternary complex. Even in the presence of latrunculin A, however, the dissociation of two proteins was too fast to calculate the binding kinetics for the actin-aplyronine A-tubulin ternary complex. 
A

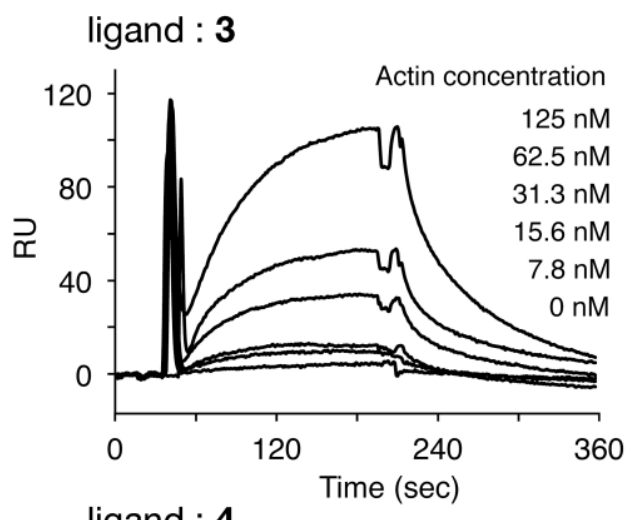

ligand : 4

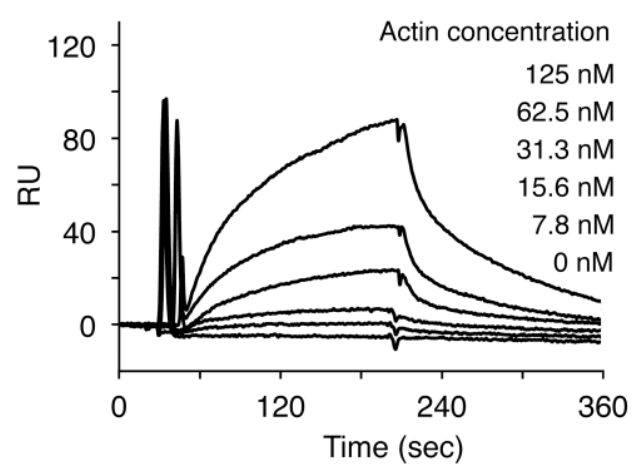

B
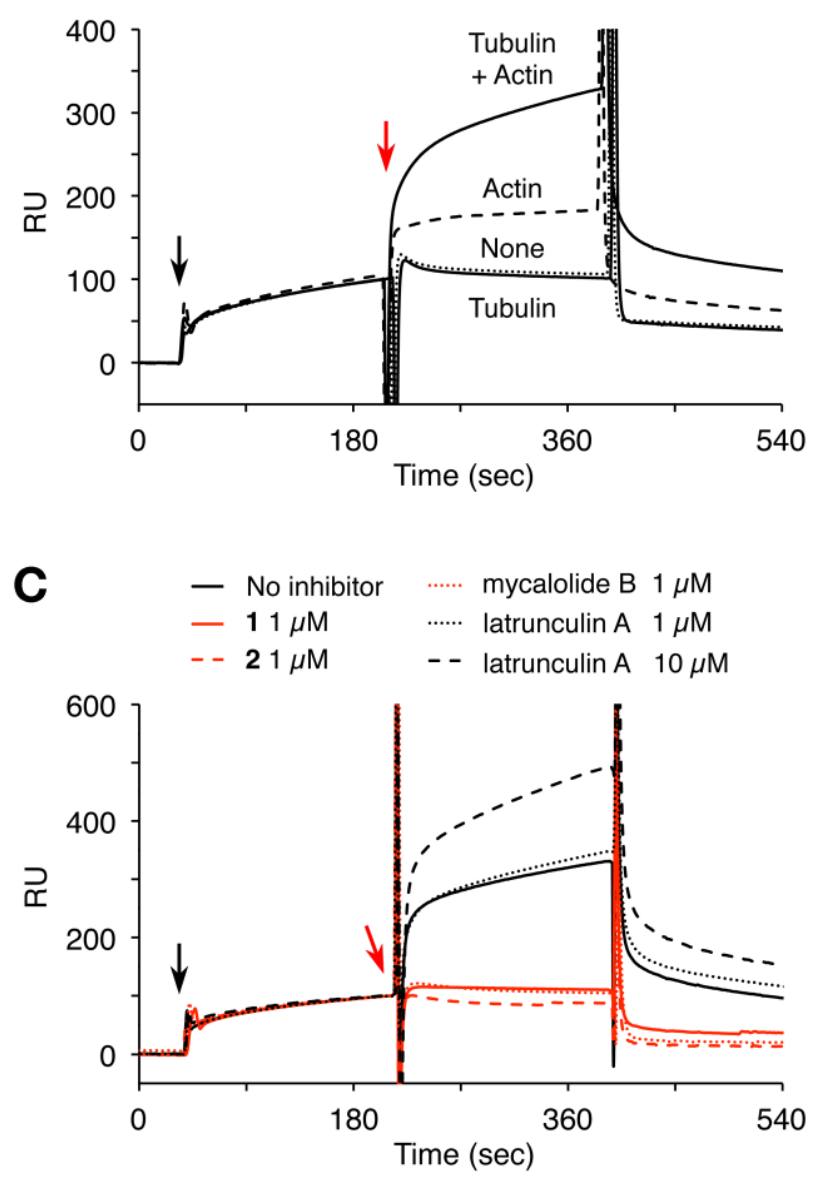

Figure 2. Interactions of actin and tubulin with immobilized aplyronines as monitored by SPR. (A) Ligands: biotin derivatives 3 and 4 . Various concentrations of actin ( 0 to $125 \mathrm{nM})$ were injected over the surface for $3 \mathrm{~min}$ in the running buffer supplemented with $10 \%$ of $10 \times$ G-buffer [final concentrations: $2 \mathrm{mM}$ Tris. $\mathrm{HCl}$ ( $\mathrm{pH} 8.0$ ), $0.2 \mathrm{mM} \mathrm{CaCl}_{2}, 0.2 \mathrm{mM}$ ATP, $0.5 \mathrm{mM}$ 2-mercaptoethanol]. After the injection, the running buffer supplemented with G-buffer was continuously introduced onto the sensor surface for $3 \mathrm{~min}$. (B, C) Ligand: biotin derivative 3 . Actin (1 $\mu \mathrm{M})$ was injected over the surface for $3 \mathrm{~min}$ in the running buffer supplemented with G-buffer (black arrow). In (B), tubulin and/or actin (1 $\mu \mathrm{M}$ each) were then continuously injected for 3 min

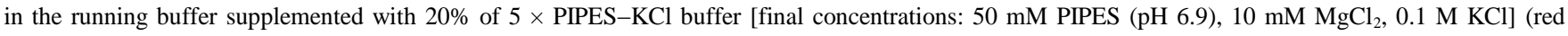
arrow). In (C), tubulin and actin (1 $\mu \mathrm{M}$ each) with various actin-depolymerizing agents were continuously injected for 3 min.

\subsection{Interaction of biotinylated tubulin with actin- aplyronine A complex}

Next, we planned to use biotinylated tubulin as a ligand for SPR analysis. To establish whether this modified tubulin specifically interacts with the actin-aplyronine A complex, an in vitro ultracentrifugation experiment was carried out. Aplyronine A (1) induced microtubule disassembly in the presence of actin, and both these proteins were dominantly detected in the supernatant (lanes 1 and 4, Figure 3A). In contrast, only actin moved to the supernatant under treatment with aplyronine $\mathrm{C}(\mathbf{2})$ (lanes 2 and 5). Under similar conditions using a 9:1 mixture of tubulin/biotinylated tubulin, both tubulin and actin moved to the supernatant under treatment with $\mathbf{1}$, as in the experiments with native tubulin. Western blotting analysis using HRP-conjugated streptavidin revealed that ca. $65 \%$ of the biotinylated tubulin was contained in the supernatant fraction, which is consistent with the results of $\mathrm{CBB}$ staining. These results suggested that actin and $\mathbf{1}$ synergistically depolymerize microtubules that contain biotinylated tubulin.

To establish the interaction of biotinylated tubulin with the actin-aplyronine A complex, we performed a gelpermeation HPLC analysis (Figure 3B). Native tubulin and biotinylated tubulin were eluted in PIPES- $\mathrm{KCl}$ buffer at almost the same time (black trace), which corresponded to the $\alpha / \beta$-tubulin heterodimer $(16.8 \mathrm{~min}, 100 \mathrm{kDa})$. When actin, $\mathbf{1}$, and a 9:1 mixture of tubulin/biotinylated tubulin were co-analyzed, two mobility peaks were detected (gray trace), which corresponded to a 1:1:1 complex of actin-aplyronine A-tubulin heterodimer (15.9 min, 145 $\mathrm{kDa})$ and the liberated actin-aplyronine A complex (18.5 min, $45 \mathrm{kDa}$ ), respectively. These elution patterns were similar to the previous experiments using native tubulin. ${ }^{6}$ 
SDS-PAGE analysis revealed that the elution of the ternary complex containing biotinylated tubulin overlapped that of native tubulin. These results strongly indicated that biotinylated tubulin can form a ternary complex with actin and $\mathbf{1}$.

\subsection{Interactions of the actin and tubulin complex with aplyronine A}

Due to the instability of diluted tubulin in vitro, biotinylated tubulin was immobilized on the streptavidin surface of a sensor chip in the presence of a 20-fold higher concentration of native tubulin in PIPES-KCl buffer (3600 3700 RU) (Figure S4). Since a significant reduction in the SPR signals was observed with the repeated injection of analyte and over time, actin and $\mathbf{1}$ were immediately coinjected after tubulin immobilization, and only the first-run sensorgram was used for each analysis. As a result, the PPI of actin and tubulin with $\mathbf{1}$ was detected in a concentrationdependent manner (Figure 4). The equilibrium constants obtained from global kinetic fitting of the sensorgrams were then fitted to an approximate 1:1 model (Figure S2). ${ }^{17}$ The on- and off-rate constants $k_{\mathrm{a}}$ and $k_{\mathrm{d}}$ of the actinaplyronine A complex to tubulin heterodimer were determined to be $4.78 \times 10^{4} \mathrm{M}^{-1} \mathrm{~s}^{-1}$ and $8.82 \times 10^{-2} \mathrm{~s}^{-1}$, respectively, and thus their dissociation constant $K_{\mathrm{D}}$ was $1.84 \times 10^{-6} \mathrm{M}$ at $25^{\circ} \mathrm{C}$. ${ }^{18}$
A

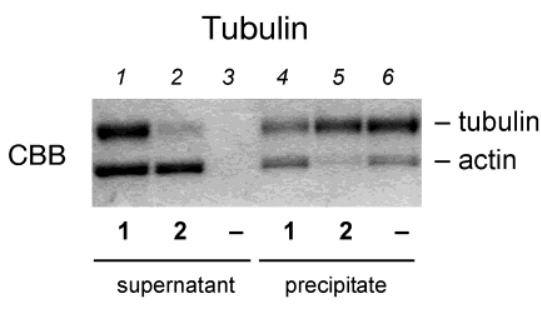

Tubulin / biotinylated tubulin (9:1)

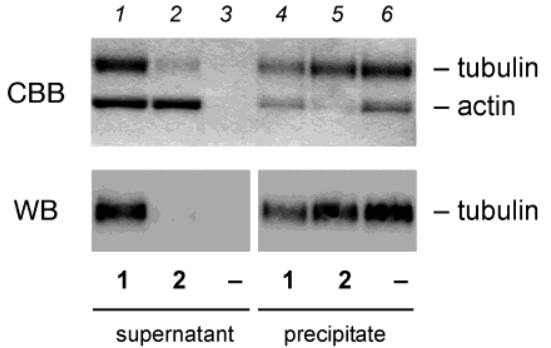

B
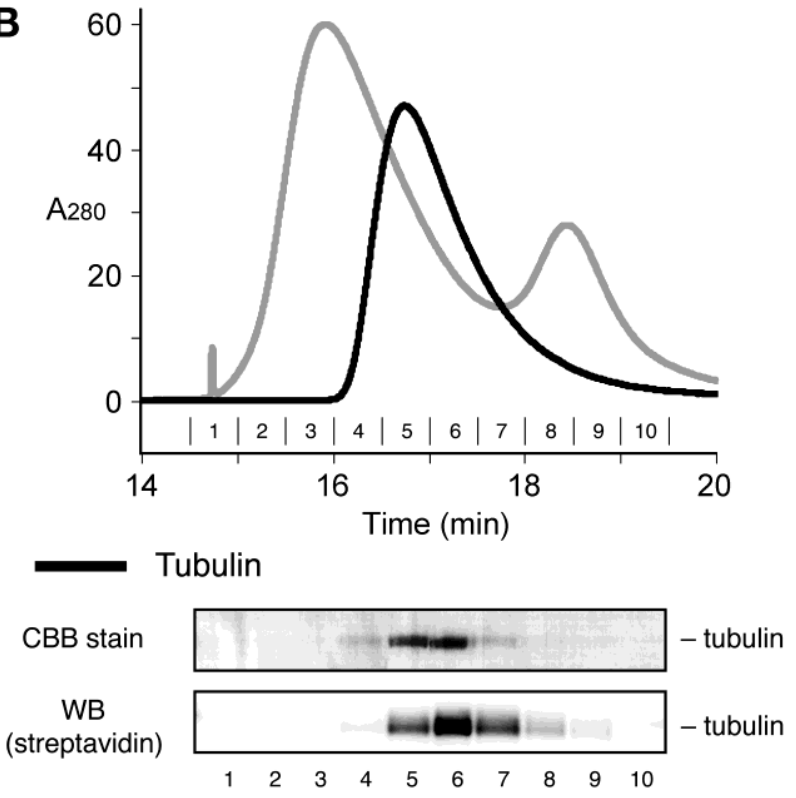

Tubulin + Actin + Aplyronine A

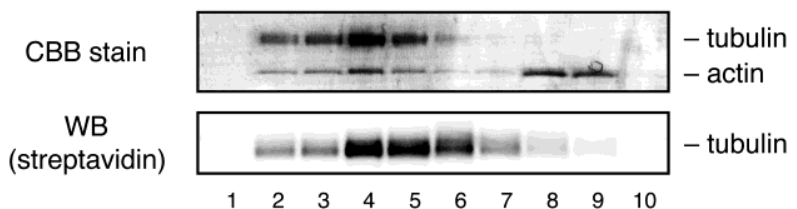

Figure 3. Interaction of biotinylated tubulin with actin-aplyronine A complex. (A) In vitro microtubule depolymerization assay. Tubulin (with or without $10 \%$ biotinylated tubulin) was polymerized with paclitaxel in the presence of actin and aplyronines, and then precipitated by ultracentrifugation. Proteins in the supernatant and the precipitate were analyzed by SDS-PAGE, and detected with CBB stain and HRP-conjugated streptavidin. (B) Gel-permeation HPLC analysis. A 9:1 mixture of tubulin/biotinylated tubulin with (gray trace) or without (black trace) actin and $\mathbf{1}$ were analyzed. Buffer, $50 \mathrm{mM}$ PIPES. $\mathrm{K}$ (pH 6.8), $10 \mathrm{mM} \mathrm{MgCl}$, $0.1 \mathrm{M} \mathrm{KCl}$; column, TSKgel SuperSW3000 ( $\phi 4.6 \times 300 \mathrm{~mm}$ ); flow rate, $0.2 \mathrm{~mL} / \mathrm{min}$; temp., $12{ }^{\circ} \mathrm{C}$; detection, UV $280 \mathrm{~nm}$. Eluted proteins (fractions 1-10) were analyzed by SDS-PAGE under reducing conditions and detected with CBB stain or HRP-conjugated streptavidin (bottom).

Previous gel-permeation HPLC studies at $8{ }^{\circ} \mathrm{C}$ showed that the association constant $K_{\mathrm{A}}$ of the actin-aplyronine A complex to tubulin heterodimer was $3.0 \times 10^{6} \mathrm{M}^{-1}$, which reflects an approximately six-fold higher affinity than this SPR analysis. This difference in affinity on these two analyses might be explained by the modification of tubulin and/or the thermal instability of the actin-aplyronine Atubulin ternary complex at $25{ }^{\circ} \mathrm{C}$ on SPR. Meanwhile, no significant binding was observed for the actin-aplyronine $\mathrm{C}$ complex with immobilized tubulin (Figure S5). These results were consistent with previous findings that the actin-aplyronine $\mathrm{C}$ complex did not attenuate microtubule 
growth in vitro, and that treatment with $\mathbf{2}$ had no detectable effects on spindle formation in tumor cells. Notably, the SPR response for the formation of a ternary complex induced by 1 was highly specific. It has not been established how aplyronine A forms the ternary complex with actin and tubulin in cells. However, in vitro assay, aplyronine A binds to actin to form the 1:1 complex. Also, only in the presence of actin, it binds to tubulin, inhibits microtubule dynamics, and forms the 1:1:1 heterotrimetric complex. Thus, we expected that aplyronine A interacts with actin prior to tubulin.

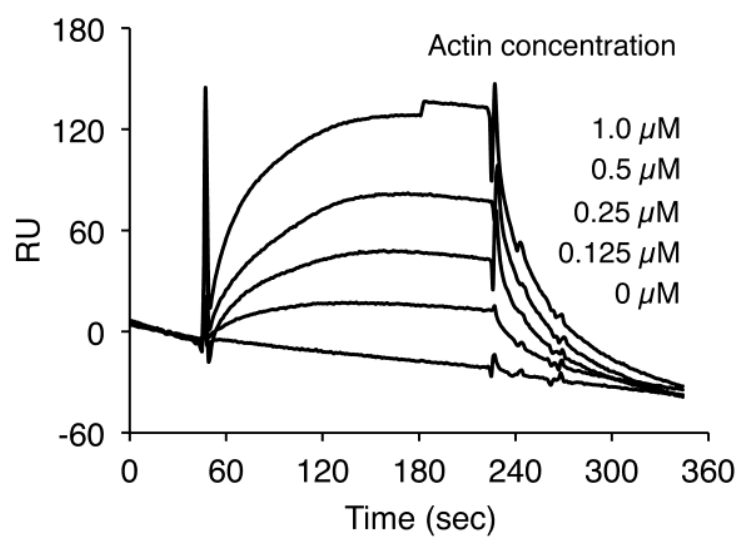

Figure 4. Interactions of the actin and tubulin complex with aplyronine A monitored by SPR. Ligand: biotinylated tubulin. Various concentrations of actin $(0$ to $1 \mu \mathrm{M})$ with $\mathbf{1}$ (2 eq. for actin) as analytes were injected over the surface for $3 \mathrm{~min}$ in PIPES-KCl buffer. After the injection, PIPES-KCl buffer was continuously introduced onto the sensor surface for $3 \mathrm{~min}$.

\section{Conclusion}

In summary, we have established the kinetics of PPI between the two polymerizing labile proteins, actin and tubulin, stabilized with an antitumor macrolide aplyronine A. SPR analysis also revealed that several actin-targeting agents specifically interfere with the PPIs including actin. Actin and tubulin are representative cytoskeleton proteins that are essential for eukaryote cellular systems. The assembly of both actin filaments and microtubules is highly regulated by numerous endogenous binding proteins. Thus, our methodology might contribute to the discovery and characterization of key compounds that regulate such multiple protein dynamics. Our study should promote further biological and pharmacological investigations of various cytoskeleton-targeting agents that show unique mechanisms of action as well as microtubule-targeting antitumor drugs.

\section{Experimental}

\subsection{General}

Aplyronine A (1) was isolated from the sea hare Aplysia kurodai, as described previously. ${ }^{1}$ Aplyronine C (2) and aplyronine A biotin conjugate (3) were prepared from natural 1. ${ }^{11,12}$ Other actin- and microtubule-targeting agents were purchased from Wako Chemical Co. unless noted otherwise. Rabbit skeletal muscle actin and biotinylated tubulin from porcine brain were purchased from Cytoskeleton Inc. Native tubulin was prepared from porcine brain using high-molarity buffer according to the literature. ${ }^{19}$ High-resolution electrospray ionization mass spectra (HR-ESIMS) were measured on an AccuTOF CS spectrometer (JEOL).

\subsection{Preparation of aplyronine $\mathrm{C}$ biotin conjugate (4)}

To a stirred solution of aplyronine C (2) $(95 \mu \mathrm{g}, 100$ $\mathrm{nmol})$ in 1,4-dioxane $(0.3 \mathrm{~mL})$ was added $2 \mathrm{M}$ aq. $\mathrm{HCl}(0.1$ $\mathrm{mL})$ under a nitrogen atmosphere. After being stirred for 80 $\min$ at $50{ }^{\circ} \mathrm{C}$, the reaction mixture was neutralized with sodium bicarbonate $(3 \mathrm{~mL})$ and extracted with chloroform $(2 \mathrm{~mL} \times 4)$. The combined extracts were washed with brine and concentrated to give aldehyde 5, which was used for the next step without further purification. To a stirred solution of $\mathbf{5}$ in methanol-acetic acid $(4: 1,0.2 \mathrm{~mL})$ was added EZ-Link Biotin-PEG ${ }_{4}$-Hydrazide $(2.5 \mu \mathrm{mol}$, Thermo Scientific) in DMSO $(10 \mu \mathrm{L})$ under a nitrogen atmosphere. After the mixture was stirred for $60 \mathrm{~h}$ at room temperature, acetone $(50 \mu \mathrm{L})$ was added. After additional stirring for 30 min, the reaction mixture was azeotropically concentrated with toluene and applied to a Develosil ODS-HG-5 reversed-phase HPLC column $(\phi 4.6 \mathrm{~mm}$ I.D. $\times 250 \mathrm{~mm}$, Nomura Chemical Co.). Samples were eluted by methanol / $40 \mathrm{mM}$ ammonium acetate $(73: 27)$ at a flow rate of 1 $\mathrm{mL} / \mathrm{min}$, with monitoring at $254 \mathrm{~nm}$, and then lyophilized from water twice to give aplyronine $\mathrm{C}$ biotin conjugate (4) (27 nmol, $27 \%$ in 2 steps, based on the HPLC analysis compared with $\mathbf{3}, t_{\mathrm{R}}=10.4 \mathrm{~min}$ ).

4.2.1. Aplyronine $\mathbf{C}$ biotin conjugate (4). HRMS (ESI) $m / z$ 1415.8582 (calcd for $\mathrm{C}_{72} \mathrm{H}_{124} \mathrm{~N}_{6} \mathrm{NaO}_{18} \mathrm{~S}[\mathrm{M}+\mathrm{Na}]^{+}, \Delta$ $-0.3 \mathrm{mmu})$.

\subsection{Cell culture and cytotoxicity assay}

HeLa S3 cells (ATCC CCL-2.2) were cultured in Eagle's minimal essential medium (E-MEM) supplemented with $10 \%$ fetal bovine serum in a humidified atmosphere containing $\mathrm{CO}_{2}(5 \%)$. Cells were seeded at $2 \times 10^{3}$ cells per well in 96-well plates. After incubation overnight at $37{ }^{\circ} \mathrm{C}$, samples in DMSO were added, and the cells were incubated for $96 \mathrm{~h}$ at $37{ }^{\circ} \mathrm{C}$. $1.4 \mathrm{mg} / \mathrm{mL} \quad 3-(4,5-$ dimethylthiazol-2-yl)-2,5-diphenyltetrazolium bromide (MTT) solution in phosphate buffer saline (PBS) $(50 \mu \mathrm{L})$ was added to the cells. After $4 \mathrm{~h}$, the culture medium was removed and the formazan product was dissolved in DMSO $(150 \mu \mathrm{L})$. Optical density at $540 \mathrm{~nm}$ was measured with a TECAN microplate reader (Infinite ${ }^{\circledR} 200$ Pro). All assays were performed in duplicate to confirm reproducibility.

\subsection{Surface plasmon resonance (SPR) analysis}

A Biacore 2000 (GE Healthcare) was used to analyze molecular interactions by means of SPR spectroscopy. All analyses were conducted at $25{ }^{\circ} \mathrm{C}$ and with a flow rate of 
$10 \mu \mathrm{L} / \mathrm{min}$ in the general running buffer [20 $\mathrm{mM}$ HEPES (pH 7.4), $0.15 \mathrm{M} \mathrm{NaCl}, 3.4 \mathrm{mM}$ EDTA, $0.05 \%$ surfactant $\mathrm{P} 20, \mathrm{pH}$ 7.4] unless noted otherwise. Biotinylated ligands or proteins were immobilized on the surface of a sensor chip SA (streptavidin). For biotinylated ligands $\mathbf{3}$ and $\mathbf{4}$, a surface density of 300 350 RU was generated by loading $10 \mu \mathrm{M}$ analytes for $1 \mathrm{~min}$. For biotinylated tubulin, a surface density of 3600 3700 RU was generated by loading $1 \mu \mathrm{M}$ biotinylated tubulin with $20 \mu \mathrm{M}$ native tubulin for 7 min in PIPES-KCl buffer with a flow rate of $15 \mu \mathrm{L} / \mathrm{min}$. Each sensorgram (time-course of the SPR signal) was corrected for the response observed in the control flow cell and normalized to a baseline of 0 RU. The association and dissociation rate constants were calculated by using Biacore 2000 Evaluation software (version 2.0.1).

\subsection{Microtubule sedimentation assay}

Tubulin ( $3 \mu \mathrm{M}$ as a heterodimer) in modified RB buffer [100 mM PIPES· Na (pH 6.9), 1 mM EGTA, 2 mM MgCl 2 ] was ultracentrifuged $\left(150,000 \times \mathrm{g}, 30 \mathrm{~min}, 4{ }^{\circ} \mathrm{C}\right)$ to remove oligomeric tubulins. To the supernatants $(500 \mu \mathrm{L})$ were added $2 \mathrm{mM}$ paclitaxel in DMSO $(0.75 \mu \mathrm{L})$ to induce microtubule formation, with or without $100 \mu \mathrm{M}$ actin in Gbuffer $(15 \mu \mathrm{L})$ and $1 \mathrm{mM}$ aplyronines $(1.5 \mu \mathrm{L})$. After incubation for $1 \mathrm{~h}$ at $37^{\circ} \mathrm{C}$, samples were ultracentrifuged $\left(150,000 \times g, 1 \mathrm{~h}, 37^{\circ} \mathrm{C}\right)$. The supernatants (lyophilized) and precipitates were dissolved in $1 \times$ SDS buffer $(100 \mu \mathrm{L})$ and boiled for $5 \mathrm{~min}$ at $95{ }^{\circ} \mathrm{C}$. SDS-PAGE was performed using a precast $10 \%$ polyacrylamide gel, and the gels were stained with a Quick-CBB kit (Wako). For immunoblot analyses, proteins in the gels after electrophoresis were transferred to PVDF membranes, using the Trans-Blot ${ }^{\circledR}$ SD semi-dry blotting system (Bio-Rad). Proteins were detected with HRP-conjugated streptavidin (1:3000, cat. no. RPN1231v, GE Healthcare). The HRP-conjugated bands were visualized with an ECL-prime system (GE Healthcare), and detected by a Fujifilm LAS-4000 MINI imaging scanner.

\subsection{Gel permeation HPLC analysis of the actin-ApA- tubulin complex}

Actin, aplyronines, and tubulin heterodimer were mixed at a 1:1:1 molar ratio $(50 \mu \mathrm{M}$ each). After incubation at room temperature for $10 \mathrm{~min}, 5 \mu \mathrm{L}$ of the mixture was analyzed by gel permeation column chromatography on a TSKgel SuperSW3000 column (TOSOH Co.), as described previously. ${ }^{6}$

\section{Acknowledgements}

We thank Prof. Takeo Usui (University of Tsukuba) for his valuable suggestions regarding the purification and biochemical experiments of tubulin. This work is supported in part by JSPS grants (25702047 to M.K. and 26242073 to H.K.) and by a Grant-in-Aid for Scientific Research on Innovative Areas from MEXT, Japan, "Chemical Biology of Natural Products." Supports were also provided by PRESTO, JST, and the Naito Foundation.

\section{Supplementary data}

Supplementary data associated with this article can be found, in the online version, at http://dx.doi.org/10.1016/j.bmc.2016.??.???.

\section{References and notes}

1. Yamada, K.; Ojika, M.; Ishigaki, T.; Yoshida, Y.; Ekimoto, H.; Arakawa, M. J. Am. Chem. Soc. 1993, 115, 11020-11021.

2. Ojika, M.; Kigoshi, H.; Ishigaki, T.; Tsukada, I.; Tsuboi, T.; Ogawa, T.; Yamada, K. J. Am. Chem. Soc. 1994, 116, 74417442.

3. Yamada, K.; Ojika, M.; Kigoshi, H.; Suenaga, K. Nat. Prod. Rep. 2007, 26, 27-43.

4. Saito, S.; Watabe, S.; Ozaki, H.; Kigoshi, H.; Yamada, K.; Fusetani, N.; Karaki, H. J. Biochem. (Tokyo) 1996, 120, 552555.

5. Hirata, K.; Muraoka, S.; Suenaga, K.; Kuroda, K.; Kato, K.; Tanaka, H.; Yamamoto, M.; Takata, M.; Yamada, K.; Kigoshi, H. J. Mol. Biol. 2006, 356, 945-954.

6. Kita, M.; Hirayama, Y.; Yoneda, K.; Yamagishi, K.; Chinen, T.; Usui, T.; Sumiya, E.; Uesugi, M.; Kigoshi, H. J. Am. Chem. Soc. 2013, 135, 18089-18095.

7. Kita, M.; Kigoshi, H. Nat. Prod. Rep. 2015, 32, 534-542.

8. Kita, M.; Kigoshi, H. J. Synth. Org. Chem. Jpn. 2015, 73, 151-160.

9. Zinzalla, G.; Thurston, D. E. Future Med. Chem. 2009, 1, 6593.

10. Milroy, L. -G.; Grossmann, T. N.; Hennig, S.; Brunsveld, L.; Ottmann, C. Chem. Rev. 2014, 114, 4695-4748.

11. Kita, M.; Hirayama, Y.; Sugiyama, M.; Kigoshi, H. Angew. Chem. Int. Ed. 2011, 50, 9871-9874.

12. Kita, M.; Hirayama, Y.; Yamagishi, K.; Yoneda, K.; Fujisawa, R.; Kigoshi, H. J. Am. Chem. Soc. 2012, 134, 20314-20317.

13. Kita, M.; Yoneda, K.; Hirayama, Y.; Yamagishi, K.; Saito, Y.; Sugiyama, Y.; Miwa, Y.; Ohno, O.; Morita, M.; Suenaga, K.; Kigoshi, H. ChemBioChem 2012, 13, 1754-1758.

14. Kuroda, T.; Suenaga, K.; Sakakura, A.; Handa, T.; Okamoto, K.; Kigoshi, H. Bioconjugate Chem. 2006, 17, 524-529.

15. Saito, S.; Watabe, S.; Ozaki, H.; Fusetani, N.; Karaki, H. J. Biol. Chem. 1994, 269, 29710-29714.

16. Wada, S.; Matsunaga, S.; Saito, S.; Fusetani, N.; Watabe, S. J. Biochem. (Tokyo) 1998, 123, 946-952.

17. The bivalent models of both tubulin heterodimer and actin for global kinetic fits were examined, but their sensorgram fitting patterns and on/off-rate constants were almost same as those of the 1:1 model. For details, see Figure S2.

18. The $K_{\mathrm{D}}$ value of actin-aplyronine A complex to tubulin heterodimer was relatively high as compared with the potent cytotoxity of aplyronine A in tumor cells. This might be due to the instability of actin and tubulin in vitro. Both proteins are needed to treat at $\mu \mathrm{M}$ or higher to maintain their physiological properties. In such a high concentration, it was impossible to promptly measure low kinetics data.

19. Castoldi, M.; Popov, A. V. Protein Expr. Purif. 2003, 32, 8388. 


\section{Graphical Abstract}

To create your abstract, type over the instructions in the template box below.

Fonts or abstract dimensions should not be changed or altered.

\begin{tabular}{|c|c|c|}
\hline Analysis of the aplyronine A-induced protein- & \multicolumn{2}{|c|}{ Leave this area blank for abstract info. } \\
\hline 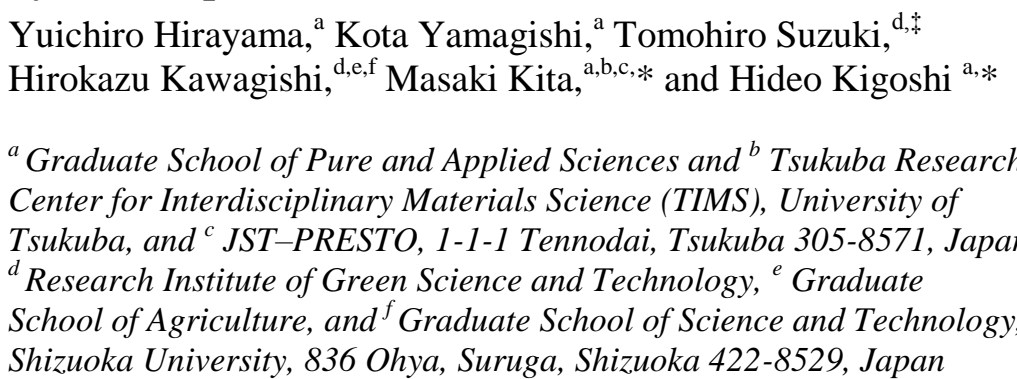 & $\begin{array}{c}\downarrow \\
\kappa_{\mathrm{D}} 4.0 \mu \mathrm{M} \\
\text { actin } \\
\text { actin-aplyronine A } \\
\text { complex }\end{array}$ & $\begin{array}{l}\text { Ligand : biotinylated tubulin } \\
\text { Analyte : actin and aplyronine A }\end{array}$ \\
\hline
\end{tabular}

